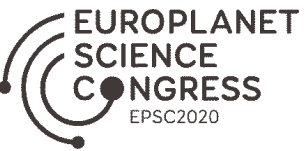

EPSC Abstracts

Vol. 14, EPSC2020-866, 2020, updated on 07 Dec 2020

https://doi.org/10.5194/epsc2020-866

Europlanet Science Congress 2020

(C) Author(s) 2020. This work is distributed under

the Creative Commons Attribution 4.0 License.

\title{
Results on stellar occultations by (307261) 2002 MS4
}

Flavia Luane Rommel ${ }^{1,2}$, Felipe Braga-Ribas ${ }^{2,3}$, Chrystian Luciano Pereira ${ }^{2,3}$, Josselin Desmars ${ }^{4,5}$, Pablo Santos-Sanz ${ }^{6}$, Gustavo Benedetti-Rossi Rossi ${ }^{2,7}$, Jose-Luis Ortiz ${ }^{6}$, Nicolás Morales ${ }^{6}$, Emmanuel Jehin $^{8}$, Julio I. B. Camargo ${ }^{1,2}$, Marcelo Assafin ${ }^{9}$, Bruno E. Morgado ${ }^{2,7}$, Roberto Vieira-Martins ${ }^{1,2}$, Bruno Sicardy ${ }^{7,10}$, Rodrigo Boufleur ${ }^{1,2}$, Alain Maury ${ }^{11}$, Joaquín Fabrega Polleri ${ }^{12}$, Peter Ceravolo ${ }^{13}$, Debra Ceravolo ${ }^{13}$, Bruce Gowe ${ }^{13}$, Rafael Sfair ${ }^{14}$, Thamiris Santana ${ }^{14}$, Luis A. Mammana ${ }^{15}$, Eduardo F. Lajus $^{16}$, Carlos A. Colazo ${ }^{17}$, Julio Spagnotto ${ }^{17}$, Áltair R. Gomes-Júnior ${ }^{2,14}$, and Othon Winter ${ }^{14}$

${ }^{1}$ National Observatory, Rio de Janeiro, Brazil (flaviarommel@on.br)

${ }^{2}$ Interinstitutional Laboratory of e-Astronomy - LIneA, Rio de Janeiro, Brazil

${ }^{3}$ Federal University of Technology - Paraná - UTFPR, Curitiba, Brazil

${ }^{4}$ Institut Polytechnique des Sciences Avancées IPSA, 63 boulevard de Brandebourg, F-94200 Ivry-sur-Seine, France

${ }^{5}$ Institut de Mécanique Céleste et de Calcul des Ephémérides, IMCCE, Observatoire de Paris, PSL Research University, CNRS,

Sorbonne Universités, UPMC Univ Paris 06, Univ. Lille, 77 Av. Denfert-Rochereau, F-75014 Paris, France

${ }^{6}$ Instituto de Astrofisica de Andalucia-CSIC, Granada, Spain

'LESIA, Observatoire de Paris, Université PSL, CNRS, Sorbonne Université, Univ. Paris Diderot, Sorbonne Paris Cité, 5 place Jules Janssen, 92195 Meudon, France

${ }^{8}$ Liège University, Belgium

${ }^{9}$ Valongo Observatory, UFRJ, Rio de Janeiro, Brazil

${ }^{10}$ PSL Research University CRNS, Sorbonne Université, UPMC Univ.Paris 06, Univ. Paris Diderot, Sorbonne Paris Cité, France

${ }^{11}$ San Pedro de Atacama Celestial Explorations - SPACE, Chile

${ }^{12}$ Panamanian Observatory in San Pedro de Atacama - OPSPA

${ }^{13}$ Anarchist Mt. Observatory, British Columbia Canada

${ }^{14}$ State University of São Paulo - UNESP, São Paulo, Brazil

${ }^{15}$ Astronomical complex El Leoncito, San Juan, Argentina

${ }^{16}$ Universidad Nacional de La Plata, La Plata (UNLP)

${ }^{17}$ Association of Argentine Observatories of Minor Bodies (AOACM)

\begin{abstract}
Transneptunian Objects (TNOs) are the remnants of our planetary system and can retain information about the early stages of the Solar System formation. Stellar occultation is a groundbased method used to study these distant bodies which have been presenting exciting results mainly about their physical properties. The big TNO called 2002 MS4 was discovered by Trujillo, C. A., \& Brown, M. E., in 2002 using observations made at the Palomar Observatory (EUA). It is classified as a hot classical TNO, with orbital parameters $\mathrm{a}=42 \mathrm{AU}, \mathrm{e}=0.139$, and $\mathrm{i}=17.70$. Using thermal measurements with PACS (Herschel) and MIPS (Spitzer Space Telescope) instruments, Vilenius et al. 2012 obtained a radius of $467+/-23.5 \mathrm{~km}$ and an albedo of 0.051 .
\end{abstract}

Predictions of stellar occultations by this body in 2019 were obtained using the Gaia DR2 catalogue and NIMA ephemeris (Desmars et al. 2015) and made available in the Lucky Star web page (https://lesia.obspm.fr/lucky-star/). Four events were observed in South America and Canada. The first stellar occultation was detected on 09 July 2019, resulting in two positives and four negatives 
chords, including a close one which proven to be helpful to constrain the body's size. This detection also allowed us to obtain a precise astrometric position that was used to update its ephemeris and improve the predictions of the following events. Two of them were detected on 26 July 2019, separated by eight hours. The first event was observed from South America and resulted in three positive detections, while the second, observed from Canada, resulted in a single chord. Another double chord event was observed on 19 August 2019 also from Canada.

Due to its size, it is expected that 2002 MS4 is in hydrostatic equilibrium. Thirouin, A. 2013 obtained a rotational light curve of 2002 MS4 and determined two possible periods ( $7.33 \mathrm{~h}$ and $10.44 \mathrm{~h}$ ) with low amplitude variation ( $0.05+/-0.01 \mathrm{mag})$. Admitting that it has a Maclaurin shape, the projected limb in the sky plane for Earth-based observers should be the same in the 09 July and 26 July events. The multi-chord detection allows determining an interval of parameters for size and shape. Considering that the same figure should have been observed in the 09 July event, we could use the both chords and the negative observations to constrain its physical parameters. With that, we could determine that 2002 MS4 has an equivalent radius of $385+/-1 \mathrm{~km}$ (Figure 1). Our results indicate that this TNO is about $100 \mathrm{~km}$ smaller in diameter than the value obtained by Vilenius et al. 2012, implying an albedo of $0.076(\mathrm{Hv}=4.0+/-0.6)$. The astrometric positions derived from these data were also helpful to improve forthcoming stellar occultations, in special the one crossing Europe on 08 August this year. More data from stellar occultations and observations of rotational light curves will help to confirm these results and assumptions.
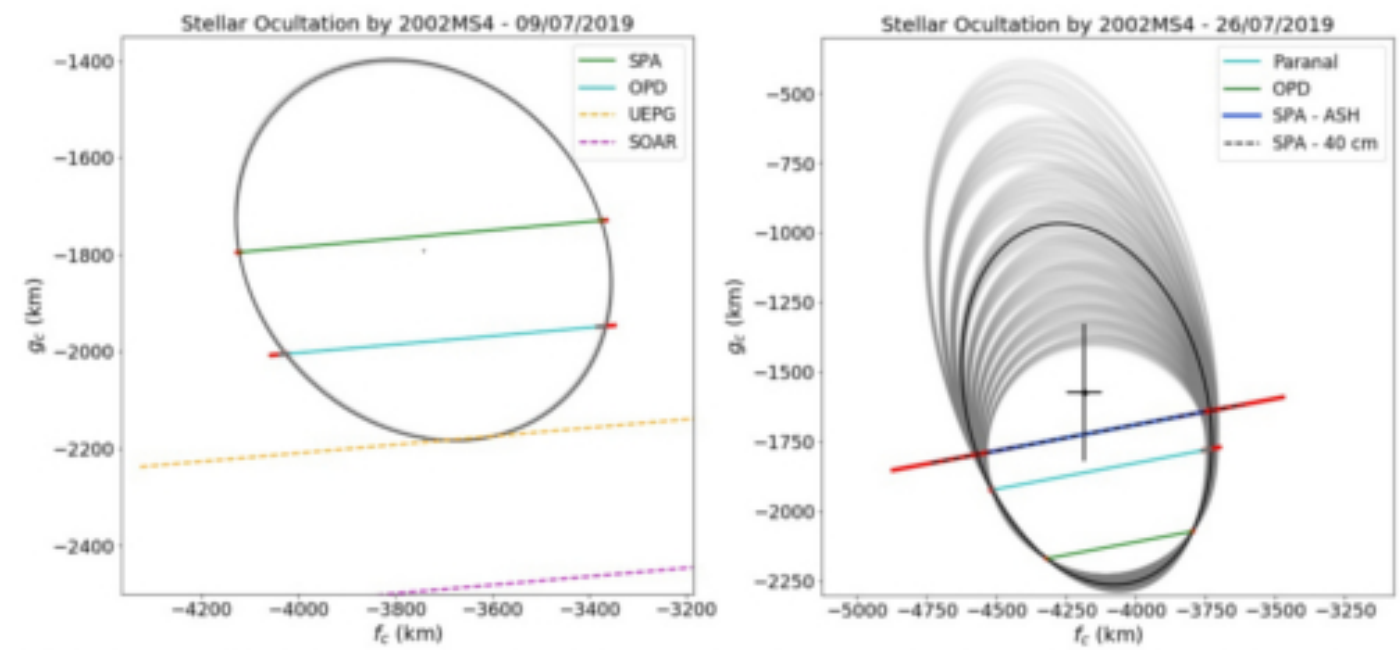

Figure 1: The positive chords are presented in blue, cyan and green, segments with error bars in red. The black ellipse represents the best solution whereas the grey ones are the possible solutions in the $1 \sigma$ error zone. The black cross represents the $1 \sigma$ uncertainty of the object's centre. On the left side the solutions for the 09 July event, constrained by the limits imposed by the 26 July event, shown on the right side. Note the negative chord from Ponta Grossa/BRA presented by the orange line, allowed constraining the ellipse solutions.

Acknowledgements: F.L.R is thankful for the support of the CAPES scholarship. The following authors acknowledge the respective CNPq grants: F.B-R 309578/2017-5; R.V-M 304544/2017-5, 401903/2016-8; J.I.B.C. 308150/2016-3; M.A 427700/2018-3, 310683/2017-3, 473002/2013-2. This study was financed in part by the Coordenação de Aperfeiçoamento de Pessoal de Nível Superior - Brasil (CAPES) - Finance Code 001 and the National Institute of Science and Technology of the e-Universe project (INCT do e-Universo, CNPq grant 465376/2014-2). G.B-R acknowledges CAPES-FAPERJ/PAPDRJ grant E26/203.173/2016, M.A FAPERJ grant E-26/111.488/2013 and A.R.GJr FAPESP grant 2018/11239-8. B.E.M thanks the CAPES/Cofecub-394/2016-05 grant. P.S-S. acknowledges financial support by the Spanish grant AYA-RTI2018-098657-J-I00 "LEO-SBNAF" (MCIU/AEI/FEDER, UE). We would like to acknowledge financial support from the State Agency for Research of the Spanish MCIU through the "Center of Excellence Severo Ochoa" award for the Instituto de Astrofisica de Andalucía (SEV-2017-0709) and the financial support by the Spanish grant AYA-2017-84637-R. Part of the results were based on observations taken at the $1.6 \mathrm{~m}$ 
telescope on Pico dos Dias Observatory of the National Laboratory of Astrophysics (LNA/Brazil). Part of this work was carried out within the "Lucky Star" umbrella that agglomerates the efforts of the Paris, Granada and Rio teams. It is funded by the European Research Council under the European Community's H2020 (2014-2020/ERC Grant Agreement No. 669416). This work has made use of data from the European Space Agency (ESA) mission Gaia (https://www.cosmos.esa.int/gaia), processed by the Gaia Data Processing and Analysis Consortium (DPAC, https://www.cosmos.esa.int/web/gaia/dpac/consortium). Funding for the DPAC has been provided by national institutions, in particular the institutions participating in the Gaia Multilateral Agreement.

\section{References}

Assafin, M. et al. PRAIA - Platform for Reduction of Astronomical Images Automatically. In: Tanga, P.; Thuillot, W. (Ed.). Gaia follow-up network for the solar system objects : Gaia FUN-SSO workshop proceedings, held at IMCCE -Paris Observatory, France, November 29 - December 1, 2010 / edited by Paolo Tanga, William Thuillot.- ISBN 2-910015-63-7, p. 85-88. [S.I.: s.n.], 2011. p. 85-88.

Desmars, J. et al. Orbit determination of trans-Neptunian objects and Centaurs for the prediction of stellar occultations. Astronomy \& Astrophysics, v. 584, p. A96, dez. 2015.

Thirouin, A. Study of Trans-Neptunian Objects using photometric techniques and numerical simulations. Dissertation. Editorial de la Universidad de Granada. Spain, 2013.

Trujillo, C. A., Brown, M. E., Minor Planet Electronic Circulars - MPEC 2002-W27. Disponível em: Jurl\{https://minorplanetcenter.net//iau/mpec/K02/K02W27.html\}.

Vilenius, E. "TNOs are cool": a survey of the trans-Neptunian region. VI. Herschel/PACS observations and thermal modelling of 19 classical Kuiper belt objects. Astronomy \& Astrophysics. v. 541, A94, 2012. 